\title{
Comparison of haemodynamic parameters between the high and low spinal block in young healthy patients
}

\author{
POREDOS P, NOVAK-JANKOVIC V
}

Department of Anaesthesiology and Intensive Care, University Medical Centre Ljubljana, Slovenia

Corresponding author:

Peter Poredos

Department of Anaesthesiology and Intensive Care

University Medical Centre Ljubljana

Zaloska 7. SI-1000 Ljubljana, Slovenia

Phone: +38615228807

Mobile: +38641252194

E-mail:peter.poredos@kclj.si

\section{ABSTRACT}

Background. For some surgical procedures a higher sensory block is needed. However, it is complicated by a higher incidence of hypotension, more bradycardia and nausea and a higher use of vasoactive drugs. In elderly and obstetric population complications have been attributed to the decrease in cardiac output and systemic vascular resistance, especially in a high block (above Th6). The aim of our study was to find the incidence of hypotension and bradycardia after a spinal anaesthesia in young, healthy patients. As young patients compensate more, we aimed to find the difference in haemodynamic variables between the group with a high and the group with a low spinal block and the underlying mechanisms of hypotension.

Methods. In a prospective, randomized study 44 American Society of Anaesthesiologists (ASA) 1 patients scheduled for knee arthroscopy under spinal anaesthesia were randomly distributed to a high (group H) and a low (group L) spinal block group. In a group $\mathrm{H}$ patients were placed into horizontal, whereas in a group $\mathrm{L}$ in 15-degree anti-Trendelenburg position immediately after the spinal block. Haemodynamic parameters were measured continuously noninvasively from 10 min before to $25 \mathrm{~min}$ after the spinal block using the CNAPTM device with the LiDCORapid monitor.

Results. The differences in haemodynamic parameters between the groups were not statistically significant at all measured times despite a significant difference in the spinal block level (18.5 vs 13.3 dermatomes above S5, p <0.001) and a significant difference in haemodynamic variables inside each group compared to the baseline value. With cardiac index (CI) as a dependent variable, a significant correlation between $\mathrm{CI}$ and stroke volume index (SVI) was found $(\beta=0.849, \mathrm{p}<0.001)$ and also between $\mathrm{CI}$ and heart rate (HR) $(\beta=0.573, p<0.001)$. In group $H$ the incidence of hypotension was $35 \%$, whereas in group L it was $10 \%$. The same difference was seen in the use of phenylephrine between the groups, however the difference was not significant.

Conclusion. In our study it was found that in young, healthy patients there are no significant differences in haemodynamic parameters and in incidence of hypotension between a high and low spinal block. Young, healthy patients compensate a decrease in systemic vascular resistance caused by the spinal anaesthesia with a compensatory increase in CI resulting from an increase in SVI and HR. However, a trend towards less hypotension, less bradycardia and less frequent phenylephrine use in a low spinal block was noted.

Key words: spinal anaesthesia, hyperbaric bupivacaine, haemodynamic parameters, cardiac output, hypotension

\section{INTRODUCTION}

Spinal anaesthesia is a safe and routinely used anaesthetic technique, however it is related to various haemodynamic changes in the patient, most commonly hypotension. $(1,2)$ The incidence of hypotension with a higher spinal block (Th7 or higher) appears to be as high as $60 \%$ or even more in elderly and obstetric population. (3-5) Hypotension may precede cardiac events
(6) and increase 1-year postoperative mortality (7) therefore, many studies have focused on the prevention of hypotension due to spinal anaesthesia. (8)

The underlying mechanism is the preganglionic sympathetic block and preserved or even increased parasympathetic nerve activity, (9) leading to a decreased systemic vascular resistance (SVR) and venodilatation, which causes a peripheral venous pooling of blood. The consequence is a lower inflow of venous blood into the heart, a decrease in cardiac output (CO) and finally a decrease in arterial blood pressure. $(10,11)$ Besides this, in higher spinal block the loss of sympathetically mediated cardiac stimulation decreases heart rate and stroke volume leading to further decreases in CO. Hypotension during a high spinal anaesthesia is thus a result of decreased CO and SVR. (12) It has been shown that even if a decrease in $\mathrm{CO}$ was prevented (with application of colloids and/or phenylephrine), hypotension could not be prevented. (13) This shows that $\mathrm{CO}$ is unable to compensate a simultaneous decrease in SVR and that the decrease in SVR could be the dominant mechanism for hypotension during a high spinal anaesthesia. (14) On the other hand, there is considerably less hypotension in patients with a lower spinal block and the decreases in systolic arterial pressure (SAP) are modest (up to 20\%). It is the consequence of the increase in sympathetic nervous system activity with a compensatory vasoconstriction in areas above the spinal block (thorax, upper limbs) (1517) and a partial increase in CO, caused by the increases in heart rate (HR) and stroke volume (SV). (18) Besides hypotension, the sympathetic block also causes brady- 
cardia, nausea, vomiting, dysrhythmias and rarely cardiac arrest. (1)

In previous studies it has been suggested that the incidence of hypotension depends on the level of spinal block and the age of the patient. $(1,3,4,19)$ The correlation between hypotension and an intrathecal dose of local anaesthetics was found with low doses resulting in less hypotension, vasopressor requirement, and nausea, presumingly because of a lower cephalic spread of local anaesthetic and a lower reduction of systemic vascular resistance. $(20,22)$ Over 60 years ago, the difference in haemodynamic parameters between a high and low spinal anaesthesia was already noticed (the limit was Th4 dermatome), but no statistical analysis was performed. (23) In a study by Asehnoune et al (19) a significant difference in the change in $\mathrm{CO}$ was seen between two groups of patients with different sensory block levels: 6th and 8th thoracic vertebra. However, the study was performed on elderly and did not show a significant difference in the incidence of hypotension. Despite the above mentioned studies, the correlation between the spinal block level and the change in haemodynamic parameters has not been sufficiently investigated. The majority of studies on haemodynamic changes in spinal anaesthesia were performed on elderly (above 60 years of age) and parturient, however their haemodynamic status may be influenced by other factors. Elderly patients have higher decreases in systemic vascular resistance during spinal anaesthesia compared to young patients, $(24,25)$ some authors consider it as the main mechanism of hypotension in elderly, (26) besides this, in the aged heart there is less of a compensatory increase in heart rate and contractility (decreased beta-adrenergic responsiveness), therefore a compensatory increase in cardiac output is smaller than in young adults or there is even a decrease in CO. On the other hand, the basal haemodynamic status of a healthy pregnant woman is characterized by a decrease in SVR, an increase in total blood volume and CO (27) and because of lower vascular tone, more blood volume is trapped in extremities. (28) Besides this, pregnant women are more susceptible to the effects of the sympathetic block. (29) Another limitation of many published studies is that the primary outcome of many studies was the incidence of hypotension, which was recognized by an intermittent non-invasive measurement of arterial blood pressure without advanced haemodynamic monitoring (CO and SVR). In this way, many episodes of hypo- tension were missed. (30)

Based on the above literature, we came to a conclusion that there is a lack of studies that would examine the haemodynamic changes during spinal anaesthesia in healthy, younger subjects, which could provide the data for a better insight into the mechanisms of hypotension and would support the efforts to prevent it.

This is the first study in young healthy nonobstetric patients that compares a high to low spinal block with the same dose of hyperbaric local anaesthetic using the continuous non-invasive recording of haemodynamic parameters. We hypothesized that different levels of the spinal block would result in significant differences in haemodynamic variables.

\section{MATERIALS AND METHODS}

In our prospective, randomized, singleblinded study 44 American Society of Anaesthesiologists (ASA) 1, patients aged between 18 and 40 years were included. Patients receiving spinal anaesthesia for knee arthroscopy between January 2014 and October 2015 were included in this study. The exclusion criteria included: chronic diseases (including peripheral arterial disease (PAD), Raynaud's syndrome and vascular surgery of the upper extremities; disorders of heart rhythm), drugs that could influence the patient's haemodynamic status, contraindications to spinal anaesthesia, patient's refusal to participate in the study, a history of allergy to local anaesthetic and a conversion to general anaesthesia.

All patients were informed about the study and gave a written consent. Premedication was performed with $7.5 \mathrm{mg}$ of oral midazolam and patients were allowed to drink clear liquids up to $2 \mathrm{~h}$ before surgery. On arrival to the operating theatre, an 18-gauge intravenous cannula was inserted in a peripheral vein on the arm, not used for measurements. Standard monitoring (pulse oximetry, ECG, oscillometric upper-arm non-invasive blood pressure) was applied and connected with a Dräger Infinity Delta monitor (Drägerwerk AG \& Co. KGaA, Lübeck, Germany). According to the risk/benefit ratio, the method of haemodynamic monitoring for a healthy patient population should be non-invasive (31) and according to availability at our institution the LiDCORapid monitor with CNAP was used. Therefore, the LiDCORapid v2 (LiDCO Ltd., Cambridge, United Kingdom) with CNAPTM monitoring system (CNSystems Medizintechnik AG, Graz, Austria) was applied on an index and middle finger on an arm without an intravenous cannula. The CNAPTM device develops an arterial waveform noninvasively by applying exterior pressure to the finger vessel wall keeping the blood volume of the finger arteries constant. The pressure in the cuff, which is needed to keep the volume constant, corresponds to arterial pressure. It is calibrated intermittently with a NIBP cuff.

The baseline measurements of haemodynamic parameters were performed after 10 minutes of lying in a supine position (systolic arterial pressure - SAP, mean arterial pressure - MAP, cardiac index - CI, systemic vascular resistance index - SVRI, stroke volume index - SVI and pulse pressure - PP). SVRI was calculated by the formula: SVRI $=80 \times($ MAP - RAP $) / C I$ (by LiDCORapid), where RAP is the right atrial pressure. A RAP value was arbitrarily set to $7 \mathrm{~mm} \mathrm{Hg}$; namely, with non-invasive methods it was not possible to measure its real value.

After the measurement, a spinal anaesthesia was performed in a sitting position at the L2-L3 or L3-4 interspace using a 26G atraumatic spinal needle with introducer (Atraucan, B. Braun Medical, Melsungen, Germany). In each patient $12,5 \mathrm{mg}$ of hyperbaric bupivacaine $0.5 \%$ (Marcaine Spinal Heavy; Astra Zeneca, Lund, Sweden) was injected intrathecally over 30 s and the patient was immediately placed in a supine position.

After anaesthetic administration, the patients were randomized into 2 groups: group $\mathrm{H}$ (high spinal block) and group $\mathrm{L}$ (low spinal block) by the method of sealed envelopes (each envelope contained a number, generated by a computer and a paper saying "high" or "low block"). All spinal blocks were performed by the same experienced anaesthesiologist. In group $\mathrm{H}$, the patient was put in a supine position with the operating table in horizontal position after the induction of subarachnoid block, whereas in group $\mathrm{L}$ the table was tilted approximately 15 degrees in anti-Trendelenburg position for 10 minutes and then into horizontal supine position. The upper sensory level of spinal block was evaluated for $10 \mathrm{~min}$ in 2-minute intervals and in 5-minute intervals for another $20 \mathrm{~min}$ until there was no change in 3 consecutive readings. It was measured as a loss of cold sensation (using a sponge immersed in icecold alcohol). The haemodynamic variables were measured constantly with a recalibration each $5 \mathrm{~min}$. A finger-cuff was switched 


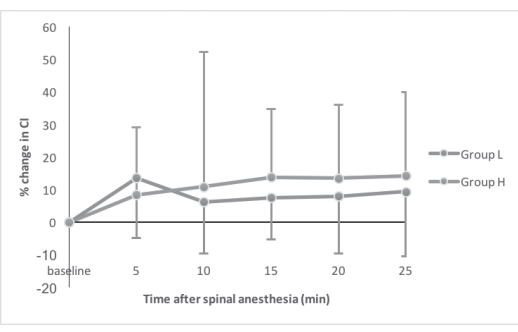

Figure 1. Time course of percentage of change in cardiac index (CI) with respect to baseline in patients with a high (group $H$ ) and patients with a low (group L) sensory block.

CI, cardiac index.

${ }^{*} \mathrm{p}<0.05$ with respect to baseline

$\dagger \mathrm{p}<0.001$ with respect to baseline

from the index to middle finger and the opposite way each $20 \mathrm{~min}$, as instructed by the CNAP company. An arm with the CNAP device was wrapped in warm swabs to prevent vasoconstriction because of low operating theatre temperatures and instructions were given to patients to keep the arm still. Time measurement started with the withdrawal of the spinal needle.

Before the induction of spinal anaesthesia all patients received $2 \mathrm{~g}$ of cefazolin in $100 \mathrm{ml}$ of saline over $15 \mathrm{~min}$, after which an infusion of lactated Ringer's solution ( $5 \mathrm{~mL} /$ $\mathrm{kg} / \mathrm{h}$ ) was started.

Hypotension was defined as SAP less than $80 \%$ of baseline - the value accepted by most investigators. (32-34) In case of hypotension, a bolus of $100 \mathrm{mcg}$ of intravenous phenylephrine was given as a rescue medication with no additional fluid. If there was concomitant bradycardia (heart rate $<50$ beats/min), a bolus of $0.5 \mathrm{mg}$ atropine was given. The anaesthesiologist performing the block and measuring the sensory block level was blinded for the haemodynamic measurements. The monitoring of the patient as well as fluid and drug requirements were managed by another anaesthesiologist, blinded for the spinal block level.

The primary objective of our study was to determine the difference in CI values and in the incidence of hypotension between the patients with a high and the patients with a low spinal block. The decision to use the $\mathrm{CI}$ as the primary variable was based on the literature showing that inadequate $\mathrm{CO}$ results in reduced organ perfusion and an impaired microcirculation. (35) The secondary outcome was to observe the incidence of complications (nausea).

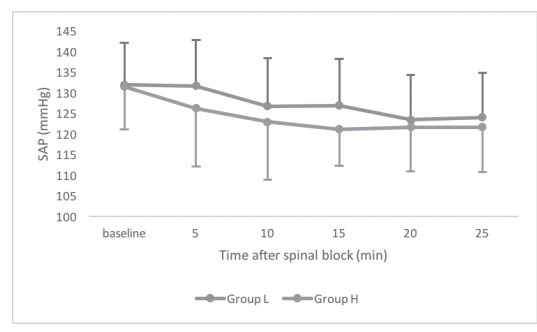

Figure 2. Time course of change in systolic arterial pressure (SAP) in patients with a high (group H) and patients with a low (group L) sensory block.

SAP, systolic arterial pressure. ${ }^{*} \mathrm{p}<0.05$ with respect to baseline $\dagger \mathrm{p}<0.001$ with respect to baseline

\section{STATISTICAL ANALYSIS}

The haemodynamic measurements were stored in the LiDCORapid monitor and downloaded as .csv files for each patient. The beat-to-beat data set was transformed into average values every $30 \mathrm{~s}$. The data for the first 3 minutes after the spinal block were inconsistent because of a change in the patient's position from sitting to a supine, therefore those data were omitted.

For the purpose of statistical analyses, dermatome levels were scored in sequence, starting at the 5th sacral segment (S5) with number 1 .

SPSS statistical program v.17.0 was used for the analysis of all data. The data were expressed as means, 95\% confidence intervals for means or as a number of patients and percentage.

For a normal distribution of the data, Kolmogorov-Smirnov test was used. The Paired Sample T-test was used to detect haemodynamic parameters' changes compared to the baseline values of the parameters, Independent Sample T-test to test the difference between the study groups and Multiple linear regression analysis to assess the relationship between the patient characteristics and baseline parameters with a change in CI, SAP, MAP, SVRI and HR. Chi-square test was used to compare the proportions of hypotension, nausea and the need for vasoactive drugs between the two groups. $\mathrm{P}<0.05$ was considered as statistically significant.

According to preliminary data and the data from the literature, (19) a sample size of 20 patients per group was determined as sufficient to detect a $25 \%$ difference in cardiac index between high and low spinal

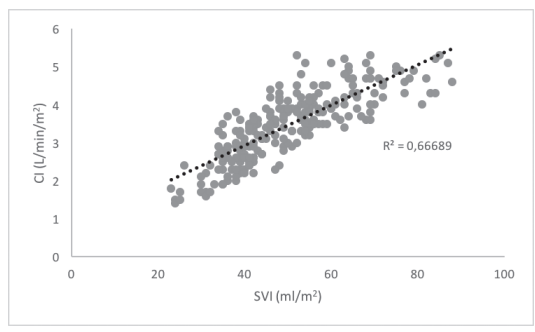

Figure 3. Scatter plot showing the correlation between the cardiac index (CI) and stroke volume index (SVI) values. The solid line represents the linear regression line.

R2 = measure of goodness-of-fit of linear regression

anaesthesia group (SD 0,9 L/min/m2) with a power of $80 \%$ and the probability level of 0.05 . To allow for a possible dropout rate of $10 \%$, we needed 44 patients in this study. The study was approved by Slovenian Medical Ethics Committee (Ref.: 175/02/10).

\section{RESULTS}

Forty-four patients referred to knee arthroscopy were randomly distributed in 2 groups receiving high or low spinal anaesthesia. One patient showed excessive movement of the fingers due to anxiety, whereas 3 additional patients were excluded from data analyses because of problems with haemodynamic monitor calibrations. Therefore, 40 patients were included in the analysis with 20 in each study group.

The variables in the majority of observed events and repeated measurements were distributed normally, the assumption that the data were distributed normally was confirmed (table 1).

Both groups had comparable demographic data (age, BMI, gender and height) and the baseline values of haemodynamic parameters (CI, SAP, MAP, SVRI, PP and HR) prior to spinal anaesthesia (table 2).

The level of sensory block was significantly higher in group $\mathrm{H}$ compared to group $\mathrm{L}$ (18.5 (95\%CI $17.9-19.1)$ vs $13.3(95 \% \mathrm{CI}$ 12.7-13.9) dermatomes above $\mathrm{S} 5, \mathrm{p}<0.001$ ) or converted into spinal segments 10th vs. 5th thoracic vertebral segment. The average time to reach the maximal level of sensory block was significantly longer in group $\mathrm{H}$ than in group L $(12.8(95 \% \mathrm{CI}$ $11.4-14.2)$ vs. 10.9 (95\%CI 10.2-11.6) $\mathrm{min}$, $\mathrm{p}=0.026)$. 
Table 1. The Kolmogorov-Smirnov and Shapiro-Wilk test of a normality of the distribution of the data in patients with high (group H) and patients with low (group L) sensory block.

\begin{tabular}{|c|c|c|c|c|c|c|c|}
\hline & \multirow[t]{2}{*}{ Group } & \multicolumn{3}{|c|}{ Kolmogorov-Smirnov } & \multicolumn{3}{|c|}{ Shapiro-Wilk } \\
\hline & & Statistic & df & Sig. & Statistic & df & Sig. \\
\hline \multirow[t]{2}{*}{ CI beginning } & $\mathrm{L}$ & ,108 & 20 &, $200^{\star}$ & ,977 & 20 & ,886 \\
\hline & $\mathrm{H}$ & ,135 & 20 &, $200^{*}$ & ,982 & 20 & ,956 \\
\hline \multirow[t]{2}{*}{$\mathrm{CI}$ after $5 \mathrm{~min}$} & $\mathrm{~L}$ &, 154 & 20 &, $200^{*}$ & 943 & 20 & 269 \\
\hline & $\mathrm{H}$ & ,175 & 20 &, 110 & ,866 & 20 &, 010 \\
\hline \multirow[t]{2}{*}{$\mathrm{CI}$ after $10 \mathrm{~min}$} & $\mathrm{~L}$ & 107 & 20 &, $200^{*}$ & ,979 & 20 & ,925 \\
\hline & $\mathrm{H}$ &, 172 & 20 &, 124 & ,958 & 20 &, 508 \\
\hline \multirow[t]{2}{*}{$\mathrm{CI}$ after $15 \mathrm{~min}$} & $\mathrm{~L}$ &, 183 & 20 & 079 & ,956 & 20 & ,473 \\
\hline & $\mathrm{H}$ &, 117 & 20 &, $200^{*}$ & 977 & 20 & ,894 \\
\hline \multirow[t]{2}{*}{$\mathrm{CI}$ after $20 \mathrm{~min}$} & $\mathrm{~L}$ &, 129 & 20 &, $200^{*}$ & 975 & 20 & ,851 \\
\hline & $\mathrm{H}$ & , 108 & 20 &, $200^{*}$ & ,963 & 20 & ,614 \\
\hline \multirow[t]{2}{*}{$\mathrm{CI}$ after $25 \mathrm{~min}$} & $\mathrm{~L}$ & ,158 & 20 &, $200^{*}$ & ,967 & 20 & ,690 \\
\hline & $\mathrm{H}$ & ,147 & 20 &, $200^{*}$ & 947 & 20 & ,320 \\
\hline \multirow[t]{2}{*}{ SAP beginning } & $\mathrm{L}$ & ,143 & 20 &, $200^{\star}$ & ,950 & 20 & ,367 \\
\hline & $\mathrm{H}$ & ,099 & 20 &, $200^{*}$ & ,980 & 20 & ,935 \\
\hline \multirow[t]{2}{*}{ SAP after 5 min } & $\mathrm{L}$ & ,151 & 20 & $200^{*}$ & 960 & 20 &, 545 \\
\hline & $\mathrm{H}$ & ,122 & 20 & $200^{*}$ & 960 & 20 &, 553 \\
\hline \multirow[t]{2}{*}{ SAP after $10 \mathrm{~min}$} & $\mathrm{~L}$ & ,088 & 20 &, $200^{\star}$ & ,985 & 20 & 983 \\
\hline & $\mathrm{H}$ & ,182 & 20 & ,082 & ,912 & 20 & 071 \\
\hline \multirow[t]{2}{*}{ SAP after $15 \mathrm{~min}$} & $\mathrm{~L}$ & ,163 & 20 & ,173 & 948 & 20 & ,336 \\
\hline & $\mathrm{H}$ &, 100 & 20 &, $200^{\star}$ & ,989 & 20 & 996 \\
\hline \multirow[t]{2}{*}{ SAP after $20 \mathrm{~min}$} & $\mathrm{~L}$ & ,090 & 20 &, $200^{*}$ & ,978 & 20 & 903 \\
\hline & $\mathrm{H}$ & , 108 & 20 &, $200^{*}$ & 951 & 20 & ,390 \\
\hline \multirow[t]{2}{*}{ SAP after $25 \mathrm{~min}$} & $\mathrm{~L}$ & ,150 & 20 &, $200^{*}$ & ,952 & 20 & ,395 \\
\hline & $\mathrm{H}$ & ,124 & 20 &, $200^{*}$ & ,978 & 20 & ,899 \\
\hline \multirow[t]{2}{*}{ MAP after 5 min } & $\mathrm{L}$ & ,159 & 20 &, $200^{*}$ & ,923 & 20 & ,115 \\
\hline & $\mathrm{H}$ & ,162 & 20 & ,179 & ,958 & 20 &, 505 \\
\hline \multirow[t]{2}{*}{ MAP after $10 \mathrm{~min}$} & $\mathrm{~L}$ &, 094 & 20 &, $200^{\star}$ & ,983 & 20 & 970 \\
\hline & $\mathrm{H}$ & ,183 & 20 & ,078 & ,906 & 20 & 053 \\
\hline \multirow[t]{2}{*}{ MAP after 15 min } & $\mathrm{L}$ & ,118 & 20 &, $200^{*}$ & ,964 & 20 & ,629 \\
\hline & $\mathrm{H}$ & 140 & 20 &, $200^{*}$ & ,966 & 20 & 677 \\
\hline \multirow[t]{2}{*}{ MAP after 20 min } & $\mathrm{L}$ &, 124 & 20 &, $200^{*}$ & ,968 & 20 & ,715 \\
\hline & $\mathrm{H}$ & ,249 & 20 & ,002 & ,904 & 20 & 049 \\
\hline \multirow[t]{2}{*}{ MAP after 25 min } & $\mathrm{L}$ &, 161 & 20 & ,189 & ,950 & 20 & ,362 \\
\hline & $\mathrm{H}$ & ,179 & 20 & ,094 & ,937 & 20 & 210 \\
\hline \multirow[t]{2}{*}{ SVRI beginning } & $\mathrm{L}$ &, 224 & 20 &, 010 &, 834 & 20 & ,003 \\
\hline & $\mathrm{H}$ & 143 & 20 &, $200^{*}$ & ,927 & 20 &, 132 \\
\hline \multirow[t]{2}{*}{ SVRI after $5 \mathrm{~min}$} & $\mathrm{~L}$ & ,173 & 20 & ,117 & ,956 & 20 & ,474 \\
\hline & $\mathrm{H}$ & ,179 & 20 & ,092 &, 878 & 20 & 016 \\
\hline SVRI after $10 \mathrm{~min}$ & $\mathrm{~L}$ & ,129 & 20 &, $200^{\star}$ & ,928 & 20 &, 141 \\
\hline & $\mathrm{H}$ &, 238 & 20 & ,004 & ,892 & 20 & 030 \\
\hline SVRI after $15 \mathrm{~min}$ & $\mathrm{~L}$ & ,175 & 20 &, 112 &, 865 & 20 & 010 \\
\hline & $\mathrm{H}$ & ,198 & 20 & ,038 &, 841 & 20 & ,004 \\
\hline SVRI after $20 \mathrm{~min}$ & $\mathrm{~L}$ &, 245 & 20 & ,003 &, 810 & 20 & 001 \\
\hline & $\mathrm{H}$ & ,161 & 20 & ,184 &, 829 & 20 & 002 \\
\hline
\end{tabular}




\begin{tabular}{|c|c|c|c|c|c|c|c|}
\hline SVRI after $25 \mathrm{~min}$ & $\mathrm{~L}$ & ,208 & 20 & ,023 & 829 & 20 &, 002 \\
\hline & $\mathrm{H}$ & ,163 & 20 & ,171 & ,853 & 20 & ,006 \\
\hline \multirow[t]{2}{*}{ SVI beginning } & $\mathrm{L}$ & 192 & 20 & ,051 & 899, & 20 & ,039 \\
\hline & $\mathrm{H}$ & 139 & 20 &, $200^{\star}$ & ,914 & 20 & 075 \\
\hline \multirow[t]{2}{*}{ SVI after $5 \mathrm{~min}$} & $\mathrm{~L}$ &, 213 & 20 &, 018 & ,830 & 20 & ,003 \\
\hline & $\mathrm{H}$ & ,082 & 20 & $200^{*}$ & 979, & 20 & 914 \\
\hline \multirow[t]{2}{*}{ SVI after $10 \mathrm{~min}$} & $\mathrm{~L}$ & 149 & 20 &, $200^{\star}$ & ,886 & 20 & ,023 \\
\hline & $\mathrm{H}$ & ,167 & 20 & ,146 & ,939 & 20 & ,229 \\
\hline \multirow[t]{2}{*}{$\underline{\text { SVI after } 15 \mathrm{~min}}$} & $\mathrm{~L}$ & 143 & 20 &, $200^{*}$ & ,915 & 20 &, 080 \\
\hline & $\mathrm{H}$ & ,089 & 20 &, $200^{\star}$ & ,965 & 20 & ,639 \\
\hline \multirow[t]{2}{*}{ SVI after $20 \mathrm{~min}$} & $\mathrm{~L}$ &, 161 & 20 & , 189 & ,939 & 20 & ,225 \\
\hline & $\mathrm{H}$ & 120 & 20 &, $200^{*}$ & ,955 & 20 & ,442 \\
\hline \multirow[t]{2}{*}{ SVI after $25 \mathrm{~min}$} & $\mathrm{~L}$ & ,152 & 20 &, $200^{*}$ & ,930 & 20 & 153 \\
\hline & $\mathrm{H}$ & ,152 & 20 &, $200^{*}$ & ,924 & 20 &, 118 \\
\hline \multirow[t]{2}{*}{ PP beginning } & $\mathrm{L}$ & ,159 & 20 & 200 & ,907 & 20 &, 055 \\
\hline & $\mathrm{H}$ & ,176 & 20 &, 106 & ,904 & 20 & ,049 \\
\hline \multirow[t]{2}{*}{$\mathrm{PP}$ after $5 \mathrm{~min}$} & $\mathrm{~L}$ & ,131 & 20 &, $200^{\star}$ & ,970 & 20 & ,758 \\
\hline & $\mathrm{H}$ & ,111 & 20 &, $200^{*}$ & ,972 & 20 &, 800 \\
\hline \multirow[t]{2}{*}{$\mathrm{PP}$ after $10 \mathrm{~min}$} & $\mathrm{~L}$ &, 114 & 20 &, $200^{*}$ & ,956 & 20 & ,465 \\
\hline & $\mathrm{H}$ &, 170 & 20 & ,131 & ,913 & 20 &, 072 \\
\hline \multirow[t]{2}{*}{$\mathrm{PP}$ after $15 \mathrm{~min}$} & $\mathrm{~L}$ & ,188 & 20 &, 061 & ,923 & 20 & ,113 \\
\hline & $\mathrm{H}$ &, 121 & 20 &, $200^{*}$ & ,960 & 20 &, 548 \\
\hline \multirow[t]{2}{*}{$\mathrm{PP}$ after $20 \mathrm{~min}$} & $\mathrm{~L}$ & ,118 & 20 &, $200^{*}$ & ,943 & 20 & ,278 \\
\hline & $\mathrm{H}$ & ,119 & 20 &, $200^{*}$ & ,968 & 20 &, 721 \\
\hline \multirow[t]{2}{*}{$\mathrm{PP}$ after $25 \mathrm{~min}$} & $\mathrm{~L}$ & ,126 & 20 &, $200^{*}$ & ,943 & 20 & ,277 \\
\hline & $\mathrm{H}$ & ,161 & 20 & ,188 & ,939 & 20 & 231 \\
\hline \multirow[t]{2}{*}{ HR beginning } & $\mathrm{L}$ & ,099 & 20 &, $200^{*}$ & ,972 & 20 &, 800 \\
\hline & $\mathrm{H}$ & ,140 & 20 &, $200^{\star}$ & ,916 & 20 & ,083 \\
\hline \multirow[t]{2}{*}{ HR after 5 min } & $\mathrm{L}$ &, 108 & 20 &, $200^{\star}$ & ,953 & 20 & 410 \\
\hline & $\mathrm{H}$ & ,100 & 20 &, $200^{*}$ & ,966 & 20 & ,678 \\
\hline \multirow[t]{2}{*}{ HR after $10 \mathrm{~min}$} & $\mathrm{~L}$ &, 164 & 20 &, 164 & ,945 & 20 & ,295 \\
\hline & $\mathrm{H}$ &, 100 & 20 &, $200^{*}$ & ,978 & 20 & 901 \\
\hline \multirow[t]{2}{*}{ HR after $15 \mathrm{~min}$} & $\mathrm{~L}$ & ,102 & 20 &, $200^{\star}$ & ,981 & 20 & ,946 \\
\hline & $\mathrm{H}$ & ,148 & 20 &, $200^{\star}$ & ,925 & 20 & 123 \\
\hline \multirow[t]{2}{*}{ HR after $20 \mathrm{~min}$} & $\mathrm{~L}$ &, 127 & 20 &, $200^{\star}$ & ,935 & 20 & 195 \\
\hline & $\mathrm{H}$ &, 100 & 20 &, $200^{*}$ & ,984 & 20 & 975 \\
\hline \multirow[t]{2}{*}{ HR after 25 min } & $\mathrm{L}$ & ,172 & 20 &, 124 & ,932 & 20 & 168 \\
\hline & $\mathrm{H}$ & ,099, & 20 &, $200^{*}$ & 972 & 20 & 804 \\
\hline
\end{tabular}

$\overline{\mathrm{CI}}=$ Cardiac index, $\mathrm{SAP}=$ systolic arterial pressure, $\mathrm{MAP}=$ mean arterial pressure, $\mathrm{SVRI}=$ systemic vascular resistance index, $\mathrm{SVI}=$ stroke volume index, $\mathrm{PP}=$ pulse pressure, $\mathrm{HR}$ = heart rate 
Table 2. The presentation of demographic, general data and baseline values of haemodynamic parameters in patients with high (group H) and patients with low (group L) sensory block. Data are presented as means (95\% confidence interval for means) or a number of patients (\%).

\begin{tabular}{llll}
\hline & Group H & Group L & p-value \\
\hline Age $($ years $)$ & $31.8(28.7-34.9)$ & $34.3(31.3-37.3)$ & 0.271 \\
\hline BMI $(\mathrm{kg} / \mathrm{m} 2)$ & $26.4(25.5-27.3)$ & $25.0(23.7-26.3)$ & 0.078 \\
\hline Sex $(\mathrm{M} / \mathrm{F})$ & $13 / 7(65 \% / 35 \%)$ & $11 / 9(55 \% / 45 \%)$ & 0.374 \\
\hline Body height $(\mathrm{cm})$ & $175.6(171.9-179.3)$ & $173.1(169.8-176.4)$ & 0.340 \\
\hline $\begin{array}{l}\text { No. of blocked dermatomes (sen- } \\
\text { sory block) above S5 }\end{array}$ & $18.5(17.9-19.1)$ & $13.3(12.7-13.9)$ & $<0.001$ \\
\hline Time to peak sensory block (min) $)$ & $12.8(11.4-14.2)$ & $10.9(10.2-11.6)$ & 0.026 \\
\hline CI (L/min/m2) & $3.14(2.82-3.46)$ & $3.27(2.88-3.66)$ & 0.603 \\
\hline SAP (mmHg) & $131.4(126.9-135.9)$ & $131.8(127.3-136.3)$ & 0.891 \\
\hline MAP (mmHg) & $96.2(92.2-100.2)$ & $98.8(94.9-102.7)$ & 0.364 \\
\hline SVRI (dyne $/ \mathrm{cm} 5^{*}$ m2) & $2457.2(2164.5-2749.9)$ & $2449.6(2079.7-2819.5)$ & 0.975 \\
\hline SVI (mL/m2/beat) & $45.1(40.9-49.3)$ & $46.0(41.2-50.8)$ & 0.772 \\
\hline PP $(\mathrm{mmHg})$ & $53.5(49.5-57.5)$ & $53.8(49.5-58.1)$ & 0.920 \\
\hline HR (beat/min) & $70.2(65.6-74.8)$ & $71.5(66.6-76.4)$ & 0.707 \\
\hline
\end{tabular}

$\mathrm{BMI}=$ body mass index, $\mathrm{CI}=$ Cardiac index, $\mathrm{SAP}=$ systolic arterial pressure, $\mathrm{MAP}=$ mean arterial pressure, $\mathrm{SVRI}=$ systemic vascular resistance index, $\mathrm{SVI}=$ stroke volume index, $\mathrm{PP}=$ pulse pressure, $\mathrm{HR}=$ heart rate

Table 3. Haemodynamic parameters' values at Baseline, 5, 10, 15, 20, and 25 minutes after the spinal anaesthesia in patients with a high (group H) and patients with a low (group L) sensory block. The data are presented as means (95\% confidence interval for means).

\begin{tabular}{|c|c|c|c|c|c|c|}
\hline & Baseline & $\begin{array}{l}5 \text { min after the } \\
\text { block }\end{array}$ & $\begin{array}{l}10 \text { min after the } \\
\text { block }\end{array}$ & $\begin{array}{l}15 \text { min after the } \\
\text { block }\end{array}$ & $\begin{array}{l}20 \text { min after the } \\
\text { block }\end{array}$ & 25 min after the block \\
\hline \multicolumn{7}{|c|}{$\mathrm{CI}(\mathrm{L} / \mathrm{min} / \mathrm{m} 2)$} \\
\hline $\begin{array}{l}\text { Group H } \\
\% \text { change }\end{array}$ & $3.14(2.82-3.46)$ & $\begin{array}{l}3.40(2.98-3.82)^{\star} \\
8.5(0.4-16.6)\end{array}$ & $\begin{array}{l}3.49(3.05-3.93)^{*} \\
11.0(4.0-18.0)^{*}\end{array}$ & $\begin{array}{l}3.58(3.18-3.98) \dagger \\
13.9(8.3-19.5) \dagger\end{array}$ & $\begin{array}{l}3.59(3.13-4.05) \dagger \\
13.6(5.9-21.3)^{\star}\end{array}$ & $\begin{array}{l}3.60(3.13-4.07)^{\star} \\
14.3(5.6-23.0)^{\star}\end{array}$ \\
\hline $\begin{array}{l}\text { Group L } \\
\% \text { change }\end{array}$ & $3.27(2.88-3.66)$ & $\begin{array}{l}3.61(3.26-3.96)^{*} \\
13.7(4.6-22.8)^{*}\end{array}$ & $\begin{array}{l}3.42(3.05-3.79) \\
6.3(-0.6-13.2)\end{array}$ & $\begin{array}{l}3.44(3.09-3.79) \\
7.6(-1.5-16.7)\end{array}$ & $\begin{array}{l}3.46(3.10-3.82) \\
8.0(-1.8-17.8)\end{array}$ & $\begin{array}{l}3.49(3.12-3.86) \\
9.4(-1.9-20.7)\end{array}$ \\
\hline \multicolumn{7}{|c|}{ SAP (mmHg) } \\
\hline $\begin{array}{l}\text { Group H } \\
\% \text { change }\end{array}$ & $131.4(126.9-135.9)$ & $\begin{array}{l}126.2(120.0-132.4)^{\star} \\
-3.9(-7.4--0.4)^{\star}\end{array}$ & $\begin{array}{l}{ }^{*} 122.9(116.7- \\
129.1)^{*} \\
-6.3\left(-10.3--2.3^{*}\right.\end{array}$ & $\begin{array}{l}121.1(117.2- \\
125.0) \dagger \\
-7.6(-10.2--5.0)^{*}\end{array}$ & $\begin{array}{l}121.6(116.9- \\
126.3) \dagger \\
-7.3(-10.1--4.5)^{*}\end{array}$ & $\begin{array}{l}121.6(116.8-126.4) \dagger \\
-7.3(-10.5--4.1)^{\star}\end{array}$ \\
\hline $\begin{array}{l}\text { Group L } \\
\% \text { change }\end{array}$ & $131.8(127.3-136.3)$ & $\begin{array}{l}131.6(126.7-136.5) \\
-0.0 \pm 6.7\end{array}$ & $\begin{array}{l}126.7(121.6- \\
131.8)^{\star} \\
3.8(1.1-6.5)^{\star}\end{array}$ & $\begin{array}{l}126.8(121.8-131.8)^{\star} \\
-3.7(-6.6--0.8)^{*}\end{array}$ & $\begin{array}{l}{ }^{*} 123.4(118.7- \\
128.1) \dagger \\
-6.2(-9.0--3.4)^{\star}\end{array}$ & $\begin{array}{l}124.0(119.3-128.7)^{\star} \\
-5.7(-8.8--2.6)^{\star}\end{array}$ \\
\hline \multicolumn{7}{|c|}{ MAP (mmHg) } \\
\hline $\begin{array}{l}\text { Group H } \\
\% \text { change }\end{array}$ & $96.2(92.2-100.2)$ & $\begin{array}{l}87.7(83.5-91.9)^{\star} \\
-8.4(-12.9--3.9)^{\star}\end{array}$ & $\begin{array}{l}85.2(80.7-89.7) \dagger \\
-11.0(-16.0--6.0)^{*}\end{array}$ & $\begin{array}{l}84.4(80.3-88.5) \dagger \\
-11.9(-16.1--7.7)^{*}\end{array}$ & $\begin{array}{l}82.9(78.8-87.0) \dagger \\
-13.6(-17.7--9.5)^{*}\end{array}$ & $\begin{array}{l}83.7(79.6-87.8) \dagger \\
-12.7(-16.6--8.8)^{*}\end{array}$ \\
\hline $\begin{array}{l}\text { Group L } \\
\% \text { change }\end{array}$ & $98.8(94.9-102.7)$ & $\begin{array}{l}92.4(87.8-97.0) \dagger \\
-6.4(-9.7--3.1)^{*}\end{array}$ & $\begin{array}{l}88.8(84.2-93.4) \dagger \\
-10.2(-13.1--7.3)^{*}\end{array}$ & $\begin{array}{l}88.8(83.7-93.9) \dagger \\
-10.1(-14.2--6.0)^{\star}\end{array}$ & $\begin{array}{l}86.6(81.7-91.5) \dagger \\
-12.4(-16.2--8.6)^{\star}\end{array}$ & $\begin{array}{l}86.2(81.2-91.2) \dagger \\
+\quad-12.7(-16.6--8.8)^{*}\end{array}$ \\
\hline \multicolumn{7}{|c|}{ SVRI (dyne $\left.e^{*} / \mathrm{cm} 5^{*} \mathrm{~m} 2\right)$} \\
\hline $\begin{array}{l}\text { Group H } \\
\% \text { change }\end{array}$ & $\begin{array}{l}2457.2(2164.5- \\
2750.0)\end{array}$ & $\begin{array}{l}2091.8(1802.4- \\
2381.2) \dagger \\
-14.4(-20.8--8.0) \dagger\end{array}$ & $\begin{array}{l}2048.6(1750.6- \\
2346.6) \dagger \\
-17.0(-22.3- \\
-11.7) \dagger\end{array}$ & $\begin{array}{l}2043.8(1738.4- \\
2349.2) \dagger \\
-17.0(-22.5--11.5) \dagger\end{array}$ & $\begin{array}{l}2016.6(1676.9- \\
2356.3) \dagger \\
\dagger-18.7(-25.0--12.4) \dagger\end{array}$ & $\begin{array}{l}2004.7(1667.8-2341.6) \\
\dagger \\
\dagger-19.3(-25.6--13.0) \dagger\end{array}$ \\
\hline
\end{tabular}




\begin{tabular}{|c|c|c|c|c|c|c|}
\hline $\begin{array}{l}\text { Group L } \\
\% \text { change }\end{array}$ & $\begin{array}{l}2449.6(2079.7- \\
2819.5)\end{array}$ & $\begin{array}{l}2016.8(1767.7- \\
2265.9)^{\star}\end{array}$ & $\begin{array}{l}2143.2(1820.3- \\
2466.1)^{\star}\end{array}$ & $\begin{array}{l}2124.6(1778.7- \\
2470.5)^{\star} \\
-12.1(-19.6--4.6)^{\star}\end{array}$ & $\begin{array}{l}2045.7(1688.6- \\
2402.8)^{\star} \\
-15.5(-23.1--7.9) \dagger\end{array}$ & $\begin{array}{l}2044.8(1674.7-2414.9)^{\star} \\
-15.7(-24.0--7.4) \dagger\end{array}$ \\
\hline \multicolumn{7}{|c|}{ SVI (mL/m2/beat) } \\
\hline $\begin{array}{l}\text { Group H } \\
\% \text { change }\end{array}$ & $45.1(40.9-49.3)$ & $\begin{array}{l}46.2(41.3-51.1) \\
3.8(-5.0-12.6)\end{array}$ & $\begin{array}{l}46.4(41.0-51.8) \\
2.8(-4.5-10.1)\end{array}$ & $\begin{array}{l}50.3(44.6-56.0)^{*} \\
11.6(4.1-19.1)^{*}\end{array}$ & $\begin{array}{l}52.5(45.7-59.3)^{*} \\
16.5(6.0-27.0)^{*}\end{array}$ & $\begin{array}{l}53.8(46.7-60.9)^{*} \\
19.4(8.0-30.8)^{*}\end{array}$ \\
\hline $\begin{array}{l}\text { Group L } \\
\% \text { change }\end{array}$ & $46.0(41.2-50.8)$ & $\begin{array}{l}51.0(45.6-56.4)^{*} \\
12.3(4.0-20.6)^{*}\end{array}$ & $\begin{array}{l}49.3(43.2-55.4)^{*} \\
7.1(0.6-13.6)^{*}\end{array}$ & $\begin{array}{l}51.5(44.9-58.1)^{\star} \\
12.0(3.7-20.3)^{\star}\end{array}$ & $\begin{array}{l}53.2(46.6-59.8) \dagger \\
15.8(6.9-24.7)^{*}\end{array}$ & $\begin{array}{l}54.2(47.2-61.2) \dagger \\
17.8(7.9-22.7)^{\star}\end{array}$ \\
\hline \multicolumn{7}{|c|}{ PP (mmHg) } \\
\hline Group H & $53.5(49.5-57.5)$ & $54.3(49.0-59.6)$ & $52.0(46.3-57.7)$ & $52.3(47.4-57.2)$ & $54.5(49.5-59.5)$ & $54.3(49.2-59.4)$ \\
\hline Group L & $53.8(49.5-58.1)$ & $60.8(56.7-64.9) \dagger$ & $57.2(52.3-62.1)$ & $57.3(52.7-61.9)$ & $56.5(51.2-61.8)$ & $57.8(52.7-62.9)$ \\
\hline \multicolumn{7}{|c|}{ HR (beat/min) } \\
\hline Group H & $70.2(65.6-74.8)$ & $73.9(68.5-79.3)^{\star}$ & $74.2(69.4-79.0)$ & $70.1(65.8-74.4)$ & $67.5(63.5-71.5)$ & $66.7(62.8-70.6)$ \\
\hline Group L & $71.5(66.6-76.4)$ & $\begin{array}{l}72.0(67.6- \\
76.4)\end{array}$ & $\begin{array}{l}70.3(65.9- \\
74.7)\end{array}$ & $\begin{array}{l}67.4(63.1- \\
71.7)^{\star}\end{array}$ & $\begin{array}{l}65.4(61.4-69.4) \\
\dagger\end{array}$ & $65.8(61.1-70.5)^{\star}$ \\
\hline
\end{tabular}

$\mathrm{CI}=$ Cardiac index, $\mathrm{SAP}=$ systolic arterial pressure, $\mathrm{MAP}=$ mean arterial pressure, $\mathrm{SVRI}=$ systemic vascular resistance index, $\mathrm{SVI}=$ stroke volume index, $\mathrm{PP}=$ pulse pressure, $\mathrm{HR}=$ heart rate

${ }^{*} \mathrm{p}<0.05$ with respect to baseline

$\dagger \mathrm{p}<0.001$ with respect to baseline

Table 4. Regression analysis with cardiac index (CI) as dependent variable and other haemodynamic parameters as independent variables.

\begin{tabular}{lllll}
\hline & B & Beta & t-value & p-value \\
\hline constant & $-3,130$ & & $-7,949$ & 0,000 \\
\hline SVRI & 0,000 & 0,022 & 0,498 & 0,622 \\
\hline SVI & 0,067 & 0,849 & 21,232 & 0,000 \\
\hline PP & 0,003 & 0,035 & 1,530 & 0,135 \\
\hline HR & 0,043 & 0,573 & 18,911 & 0,000 \\
\hline
\end{tabular}

$\overline{\mathrm{CI}}=$ Cardiac index, $\mathrm{SVRI}=$ systemic vascular resistance index, $\mathrm{SVI}=$ stroke volume index, $\mathrm{PP}=$ pulse pressure, $\mathrm{HR}=$ heart rate

Table 5. The frequency distribution of hypotension, bradycardia, atropine and ephedrine consumption in patients with a high (group H) and patients with a low (group L) sensory block. The data are presented as number of patients (\%).

\begin{tabular}{llll}
\hline & Group H $(\mathbf{n}=\mathbf{2 0})$ & Group L $(\mathbf{n}=\mathbf{2 0})$ & p-value \\
\hline Hypotension & $7(35 \%)$ & $2(10 \%)$ & 0.064 \\
\hline Bradycardia & 3 & 0 & 0.115 \\
\hline Phenylephrine requirement & $7(35 \%)$ & $2(10 \%)$ & 0.064 \\
\hline Atropine requirement & 3 & 0 & 0.115 \\
\hline Nausea & $5(25 \%)$ & $1(5 \%)$ & 0.091 \\
\hline
\end{tabular}

The primary outcome in our study was the change in CI values after different levels of spinal anaesthesia. In group $\mathrm{H}$ the values of CI were significantly higher at all measurement periods $(5,10,15,20$ and $25 \mathrm{~min})$ after the spinal block compared to the baseline value (table 3 , figure 1 ). To contrast, a significant increase in CI was seen only 5 minutes after the spinal block in group $\mathrm{L}$ with respect to the baseline value, whereas at 10, 15, 20 and 25 minutes after the block the increase in CI compared to baseline was not significant. At all times the values of CI were above baseline in both groups. No significant difference in CI was found between the groups at any time $(p=0.946)$ (table 3).

A significant decrease in a SAP was seen at all times after the spinal block in group $\mathrm{H}$ compared to the baseline value, whereas in group L the decrease was significant at $10 \mathrm{~min}$ or later after the spinal block (table 3, figure 2). Comparing both groups of patients, no significant difference in SAP change with respect to the baseline values was found between the groups at any time $(\mathrm{p}=0.289)$. Concomitantly, a significant 
decrease in MAP and SVRI values with respect to baseline was seen in both groups at all measurements. As for SVI, there was a significant increase in SVI values at 15 minutes or later after the block in group $\mathrm{H}$ and at all measured times in group L. In no patient a MAP value $<60 \mathrm{mmHg}$ was recorded. Heart rate values were significantly increased compared to baseline only 5 minutes after the block in group $\mathrm{H}$, later the difference was not significant. In group $\mathrm{L}$ a significant decrease in $\mathrm{HR}$ was seen at 15 minutes or later after the block. Comparing both studied groups, no significant difference was found in any of the measured haemodynamic parameters between the groups at any time.

With independent variables (SVRI, SVI, $\mathrm{PP}, \mathrm{HR}$ ) we were able to explain $98.7 \%$ of $\mathrm{CI}$ as a dependent variable $(\mathrm{R} 2=0.987$, $\mathrm{R}=0.993, \mathrm{p}<0.001)$. A significant correlation between CI and SVI was found $(\beta=0.849, p<0.001)$ (figure 3$)$ and also between CI and HR $(\beta=0.573, p<0.001)$ (table 4). With other haemodynamic parameters no significant correlation was found, also no correlation was found with the level of sensory block $(\mathrm{R} 2=0.006, \mathrm{R}=0.077$, $\mathrm{p}=0.622$ ). With SAP as a dependent variable, a significant correlation was only found between SAP and pulse pressure ( $22=0.681, \mathrm{R}=0.825, \mathrm{p}=0.004, \mathrm{~B}=0.686$, $\beta=0.738, p<0.001$ ), with MAP there was no correlation. Also, no significant correlation between the decrease in SAP and the level of sensory block was found ( $R 2=0.001$, $\mathrm{R}=0.032, \mathrm{p}=0.845$ ).

The overall incidence of hypotension in our study was $22,5 \%$ ( $9 / 40$ patients), with $35 \%(7 / 20)$ in group $\mathrm{H}$ and $10 \%(2 / 20)$ in group L, however the difference between the groups was not significant ( $\mathrm{p}=0.064$ ). All patients with hypotension received 1 bolus of $100 \mathrm{mcg}$ of phenylephrine, resulting in 7 doses of phenylephrine in group $\mathrm{H}$ and 2 in group L, again the difference between the groups was not significant $(\mathrm{p}=0.064)$. A significant correlation between the phenylephrine consumption and sensory block level was found (Pearson correlation coefficient $(\rho)=0.367$, $\mathrm{p}=0.020$ ). There was a trend of higher incidence of nausea in group $\mathrm{H}$, however the difference was not significant (table 5). No patient vomited during the study.

\section{DISCUSSION}

In this prospective randomized study, we found no significant difference in haemodynamic parameters between high and low spinal anaesthesia in young, healthy subjects, with which our hypothesis was disproved. However, there was a trend of advanced haemodynamic instability in patients with a high spinal block. Also, the differences in the incidence of hypotension were not statistically significant between the groups of patients.

To our knowledge, this is the first study that studied the influence of spinal anaesthesia level on haemodynamic variables in healthy, young, non-pregnant participants using non-invasive, continuous measurement method. Both study groups were similar in terms of age, gender, height, body mass index (BMI), time to maximal sensory level of spinal block and baseline values of haemodynamic parameters.

The level of sensory block was significantly higher in group $\mathrm{H}$ compared to group L, which was accomplished with the use of the same dose of hyperbaric bupivacaine $(12,5 \mathrm{mg})$ in both groups. The difference in the level of sensory block was achieved by the tilt of the operating table with a slight anti-Trendelenburg position in group L. It was shown before that with the positioning of the patient, different levels of sensory block could be achieved. (36-38) We decided for the dose of hyperbaric bupivacaine that is high enough to produce high spinal block $(12,5 \mathrm{mg})$, even in young patients. Namely, in young patients, the sensory block level after subarachnoid injection of hyperbaric local anaesthetic solution is usually 3-4 spinal segments lower than in elderly. $(19,39,40)$ Hyperbaric bupivacaine was chosen as it is easier o get the reliable spinal block level. (41) In our patients (in group $\mathrm{H}$ ) a higher spinal block was applied as would be required by the surgical procedure, however it was performed on ASA 1 population with a normal compensatory reserve, patients who could well tolerate haemodynamic shifts caused by high spinal anaesthesia.

In most studies, different levels of spinal block were achieved through different dosages of local anaesthetic $(19-22,42,43)$ and/or different baricity of local anaesthetics. (44) The conclusion of majority of studies was that with a lower dose of the local anaesthetic patients were more haemodynamically stable and that it was most probably the consequence of a different level of sympathetic block. $(45,46)$ The drawback of lowering the doses $(<5 \mathrm{mg})$ was the increasing incidence of spinal block failures with pain, slower onset and shorter duration of the block and also the increasing rate of conversions into general anaesthesia. $(42,44,47,48)$ In contrast to the above mentioned studies, Langesaeter et al. (20) showed that there was a difference in the incidence of hypotension with the same sensory block level, but a different local anaesthetic dosage. According to the above described findings, the focus of our study was the level of spinal block as the underlying factor for the difference in haemodynamic parameters during a spinal anaesthesia excluding different doses and/ or baricities of local anaesthetic.

A mean time to maximum level of sensory block was 12.8 minutes for group $\mathrm{H}$ and 10.9 minutes for group L. The timings are comparable to previous studies with a mean time of 15 minutes and a range 11-20 minutes. $(49,50)$

The primary outcome of our study was the difference in CI value change between the group of patients with a high and the group with a low spinal block. CI is a primary determinant of global oxygen transport from the heart to the body, (51) therefore it was chosen as a primary outcome. In group $\mathrm{H}$ a significant increase in CI was found at all times. In group L there was a significant increase in CI values only 5 minutes after the block. Because of a lower block of sympathetic nerves in group L less compensatory increase in CI was necessary leading to insignificantly higher values of CI (above baseline) in this group 10 minutes or more after spinal anaesthesia. CI values in all patients were above baseline at any time after the spinal block. Also, in the study by Dyer et al. (18) it was shown that there is an increase in CI during a spinal anaesthesia, which could be caused by the increases in HR and SV. These findings were confirmed by our study, where a significant correlation between an increase in CI and an increase in SV and a correlation between an increase in the CI values and an increase in HR was found. The most probable reason for a CI increase is compensatory response to a significant decrease in a SVRI value (at the same time SVI increased significantly), but it could also be attributed to the change of the patient's position from sitting to supine. What is more, the haemodynamic curves showed the most prominent decrease in SVRI with a concomitant increase in $\mathrm{CI}$ in the first 5 minutes after the spinal block in group $\mathrm{H}$ and group $\mathrm{L}$, with a peak effect after approximately 4 minutes. Similar findings were published by Langesaeter et al., (20) who found maximal change in cardiac output and systemic vascular resistance 3 minutes after the spinal block, however, in their study a lower dose of isobaric bupivacaine was used.

When we compared the increase in $\mathrm{CI}$ values between group $\mathrm{H}$ and group $\mathrm{L}$, no significant difference was noted at all 
measured times. Most probably the lack of significance between the groups was the consequence of strong compensatory mechanisms (vasoconstriction) in the regions above the block and an increase in SVI and HR, which is more prominent in young patients. Compensatory vasoconstriction was probably more prominent in group $\mathrm{H}$, however, we did not measure the sympathetic activity (e.g. by heart rate variability) to prove the theory. (17) Another possible factor could be the insufficient difference in spinal block level to show the difference in haemodynamic parameters in young, healthy patients, also, the block was not high enough to prevent a compensatory vasoconstriction in the non-blocked areas. Besides this, all episodes of hypotension were promptly treated, preventing significant changes from happening. Our findings are in contrast to Asehnoune, who found significant difference in changes of $\mathrm{CO}$ values between the group of patients with the spinal block up to Th6 and the group up to Th8. However, their study was performed on elderly and also ASA 2 patients. It has been shown before that elderly patients have larger decreases in systemic vascular resistance during spinal anaesthesia compared to young patients $(24,25)$ and a decreased beta-adrenergic responsiveness.

Systolic arterial pressure and MAP decreased significantly after the spinal anaesthesia in both treatment groups, only in group L the decrease in SAP was not significant 5 minutes after the spinal block. However, the difference between the groups in the change of SAP and MAP values was not significant at any time. No episode of MAP below $60 \mathrm{mmHg}$ was recorded. These results show that despite the significant increase in CI, the decrease in SVRI could not be compensated for and the consequent drop in SAP and MAP could not be prevented, except in group L 5 minutes after the spinal block. The decrease in SAP $>20 \%$ (hypotension) was found in $22.4 \%$ patients, higher in group $\mathrm{H}(35 \%)$ compared to group L (10\%), however, the difference was not statistically significant. In each subject with hypotension there was only 1 episode of hypotension, responsive to vasoactive drugs. The overall incidence of hypotension was low, which could be attributed to a relatively low block and compensatory mechanisms in young subjects. In a recent study Lawicka et al. (52) showed that in patients aged around 40-years of age ASA status 1 and 2, the incidence of hypotension with the spinal block around 6th thoracic segment was 39\%. The incidence of hypotension was similar to our group $\mathrm{H}$ in which spinal block level was around Th4, although a much lower dose of hyperbaric bupivacaine was used in our study. It suggests that hypotension could depend on spinal anaesthesia level, despite different doses of local anaesthetic. Carpenter et al. (1) defined the spinal block above the level of 5th thoracic vertebra as the risk factor for hypotension in non-obstetric population. The studies on obstetric population showed an incidence of hypotension as high as $81 \%, 53$ 56) which was the consequence of a high spinal block (above Th6 or even Th4) and higher susceptibility of pregnant women to the effects of sympathetic block. (29)

The incidence of bradycardia in our study was $7.5 \%$ with bradycardia only in group $H$. The incidence was lower than in the study by Lesser et al., (57) because in our study there was no bradycardia in group $\mathrm{L}$. If we took into account only group $\mathrm{H}$, the incidence was $15 \%$, which was higher than published before. This was most probably the consequence of a higher spinal block, the average patients' age below 37 years, ASA1 and elective surgery, risk factors acknowledged also by other authors. $(1,57)$ The initial increase in HR 5 minutes after the block was most probably a compensatory response to a decrease in SVRI and it was probably also the consequence of a diminished parasympathetic activity to the heart. $(9,18)$ Cook et al. (9) showed an initial increase in HR of 6-8\% after the spinal block, which was higher than in our study (5\%). In group L, a significant decrease in HR occurred after 15 min, which matched the time to the maximal spinal block and was the consequence of a sympathetic block.

All patients in our study received only a maintenance infusion of crystalloids to avoid the influence of fluids on haemodynamic parameters and to decrease the probability of urine retention. Despite this, the incidence of hypotension was low and it was treated with phenylephrine. The decision for the phenylephrine was based on the mechanism of hypotension (the decrease in SVR) and the safety and efficacy of the drug. (58) With the use of phenylephrine boluses, it was easier to keep the values of haemodynamic parameters closer to baseline values, (59) as it had a faster onset and could be more accurately titrated than other vasoactive drugs. (60)

Besides hypotension, other common sideeffects related to hypotension were nausea, vomiting, and dizziness with incidences of around $25 \%$. (61) In our study, the incidence of nausea in group $\mathrm{H}$ was $25 \%$, whereas in group L it was $5 \%$, the differ- ence between the groups was not significant.

Haemodynamic parameters in our study were measured using CNAPTM (continuous non-invasive arterial pressure) in combination with a LiDCO Rapid monitor. It was shown before that cardiac output and stroke volume were more valuable for detecting haemodynamic changes than conventional non-invasive monitoring (NIBP). (62) The CNAPTM device has been used as arterial pressure monitoring in patients with a spinal anaesthesia before (63) and it has detected more hypotensive episodes after a spinal anaesthesia than intermittent blood pressure devices (NIBP). Besides, a rigorous anaesthesia clinical trial showed real time estimates of arterial pressure by CNAP were comparable with an invasive arterial pressure, (64) a gold standard of invasive measurements. In spontaneous breathing, low-risk patients invasive monitoring using arterial line would not be ethically justified. (65) According to our experience, a CNAP device has failures in recordings and needs recalibration more often than the 20-min intervals, advised by the manufacturer. We also followed the advice from the company to synchronize a NIBP cuff of the main patient monitor with the CNAP device. The PulseCO algorithm that was used in analysis of arterial pressure curve by LiDCORapid monitor, has been validated and used in healthy, pregnant patients receiving spinal anaesthesia. $(66,67)$ The absolute value of $\mathrm{CI}$ measured with this method has been controversial, however its value for monitoring the trends in CI values has been validated and accepted, also in spinal anaesthesia. $(30,63,68)$ We were aware of the limitations of the method used in the assessment of absolute values of CI; also in SVRI calculations the mean atrial pressure was not measured but arbitrarily set to 7 $\mathrm{mmHg}$.

The strengths of our study: after a rigorous literature review, it appears that this is the first prospective randomized study on spinal anaesthesia in young, healthy patients with two different levels of spinal block. The study will certainly add to knowledge and will form the basis for future research in risk factors for hypotension during spinal anaesthesia and its prevention.

Our study had several limitations. The method used to measure haemodynamic parameters (CNAP) was susceptible to patient movement and low operating theatre air temperature. Besides this, the patient position was changed from sitting to supine after spinal anaesthesia influencing 
haemodynamic measurements in the first 5 minutes. Additionally, patients in group L were tilted anti-Trendelenburg position for the first 10 minutes probably causing more prominent compensatory vasoconstriction in the areas above the spinal block. The study involved a relatively small number of patients (it was powered to the difference in CI and not the other variables), which may impact the generalizability of the results. Also, it was only single blinded, as the researcher could not be blinded to block level, which adds to selection bias.

\section{CONCLUSIONS}

In young, healthy patients no significant difference was found in haemodynamic parameters between a group with a high and a group with a low level of sensory block using the same dose of hyperbaric bupivacaine. However, a trend towards less hypotension, less bradycardia and less frequent phenylephrine use in low spinal block was seen. This study proves that the main mechanism of hypotension in spinal anaesthesia is the decrease in systemic vascular resistance, however, young, healthy patients compensate much higher levels of spinal anaesthesia than other population. A possible strategy for reducing spinal anaesthesia induced hypotension and other haemodynamic deterioration according to our study still remains to minimize the peak block level to as low as possible for the planned procedure, which can be achieved only by adjusting the patient position. However, determining the most appropriate preventive measure and finding the risk factors for hypotension in young, healthy patients remains to be further studied.

\section{REFERENCES}

1. Carpenter RL, Caplan RA, Brown DL et al. Incidence and risk factors for side effects of spinal anesthesia. Anesthesiology 1992; 76: 906-916

2. Critchley LA, Stuart JC, Short TG et al. Haemodynamic effects of subarachnoid block in elderly patients. British journal of anaesthesia 1994; 73: 464-470

3. Coe AJ, Revanas B. Is crystalloid preloading useful in spinal anaesthesia in the elderly? Anaesthesia 1990; 45: 241-243

4. Tarkkila PJ, Kaukinen S. Complications during spinal anesthesia: a prospective study. Regional anesthesia 1991; 16: 101-106

5. McCrae AF, Wildsmith JA. Prevention and treatment of hypotension during central neural block. British journal of anaesthesia 1993; 70: $672-680$

6. Sprung J, Warner ME, Contreras MG et al. Predictors of survival following cardiac arrest in patients undergoing noncardiac surgery: a study of 518,294 patients at a tertiary referral center. Anesthesiology 2003; 99: 259-269

7. Monk TG, Saini V, Weldon BC et al. Anesthetic management and one-year mortality after noncardiac surgery. Anesthesia and analgesia 2005; 100: 4-10 DOI: 10.1213/01.ANE.0000147519.82841.5E

8. Talakoub R, Fani A, Hirmanpour A. Comparison of the effects of colloid preload, vasopressor administration and leg compression on hemodynamic changes during spinal anesthesia for lumbar disc surgery in knee-chest position. Advanced biomedical research 2015; 4: 181 DOI: 10.4103/2277-9175.164002

9. Cook PR, Malmqvist LA, Bengtsson M et al. Vagal and sympathetic activity during spinal analgesia. Acta anaesthesiologica Scandinavica 1990; 34: 271-275

10. Riley ET. Editorial I: Spinal anaesthesia for Caesarean delivery: keep the pressure up and don't spare the vasoconstrictors. British journal of anaesthesia 2004; 92: 459-461 DOI: 10.1093/bja/aeh084

11. Azzolina R CG, Navarria D, Chiarenza F, Fiorino LS, Francesco AD, et al. Current guldelines for the prevention of hypotension induced by spinal anesthesia. . Acta Medica Mediterranea 2011;27: 107

12. Hirose M, Chun TY, Tobita M et al. The influence of aging on skin temperature and hemodynamic changes during spinal anesthesia. Acta anaesthesiologica Scandinavica 1995; 39: 891-895

13.Zorko N, Kamenik M, Starc V. The effect of Trendelenburg position, lactated Ringer's solution and 6\% hydroxyethyl starch solution on cardiac output after spinal anesthesia. Anesth Analg 2009; 108: 655-659 DOI: 10.1213/ane.0b013e31818ec9e5

14. Rooke GA, Freund PR, Jacobson AF. Hemodynamic response and change in organ blood volume during spinal anesthesia in elderly men with cardiac disease. Anesth Analg 1997; 85: 99-105

15. Arndt JO, Hock A, Stanton-Hicks M et al. Peridural anesthesia and the distribution of blood in supine humans. Anesthesiology 1985; 63: 616-623

16. Ledowski T, Paech MJ, Browning R et al. An observational study of skin conductance monitoring as a means of predicting hypotension from spinal anaesthesia for caesarean delivery. International journal of obstetric anesthesia 2010; 19: 282-286 DOI: 10.1016/j. ijoa.2010.01.001

17. Hanss R, Ohnesorge $\mathrm{H}$, Kaufmann $\mathrm{M}$ et al. Changes in heart rate variability may reflect sympatholysis during spinal anaesthesia. Acta anaesthesiologica Scandinavica 2007; 51: 1297-1304 DOI: 10.1111/j.1399-6576.2007.01455.x

18. Dyer RA, Reed AR, van Dyk D et al. Hemodynamic effects of ephedrine, phenylephrine, and the coadministration of phenylephrine with oxytocin during spinal anesthesia for elective cesarean delivery. Anesthesiology 2009; 111: 753-765 DOI: 10.1097/ ALN.0b013e3181b437e0

19. Asehnoune K, Larousse E, Tadie JM et al. Small-dose bupivacaine-sufentanil prevents cardiac output modifications after spinal anesthesia. Anesthesia and analgesia 2005; 101: 1512-1515 DOI: 10.1213/01.ANE.0000180996.91358.CC

20. Langesaeter E, Rosseland LA, Stubhaug A. Continuous invasive blood pressure and cardiac output monitoring during cesarean delivery: a randomized, double-blind comparison of low-dose versus high-dose spinal anesthesia with intravenous phenylephrine or placebo infusion. Anesthesiology 2008; 109: 856-863 DOI: 10.1097/ALN.0b013e31818a401f

21. Glaser C, Marhofer P, Zimpfer G et al. Levobupivacaine versus racemic bupivacaine for spinal anesthesia. Anesthesia and analgesia 2002; 94: 194-198, table of contents

22. Minville V, Asehnoune K, Delussy A et al. Hypotension during surgery for femoral neck fracture in elderly patients: effect of anaes- 
thetic techniques. A retrospective study. Minerva anestesiologica 2008; 74: 691-696

23. Mueller RP, Lynn RB, Sancetta SM et al. Studies of hemodynamic changes in humans following induction of low and high spinal anesthesia. II. The changes in splanchnic blood flow, oxygen extraction and consumption, and splanchnic vascular resistance in humans not undergoing surgery. Circulation 1952; 6: 894-901

24. Mark JB, Steele SM. Cardiovascular effects of spinal anesthesia. International anesthesiology clinics 1989; 27: 31-39

25. Hamel MB, Henderson WG, Khuri SF et al. Surgical outcomes for patients aged 80 and older: morbidity and mortality from major noncardiac surgery. Journal of the American Geriatrics Society 2005; 53: 424-429 DOI: 10.1111/j.1532-5415.2005.53159.x

26. Nakasuji M, Suh SH, Nomura M et al. Hypotension from spinal anesthesia in patients aged greater than 80 years is due to a decrease in systemic vascular resistance. Journal of clinical anesthesia 2012; 24: 201-206 DOI: 10.1016/j.jclinane.2011.07.014

27. Clapp JF, 3rd, Capeless E. Cardiovascular function before, during, and after the first and subsequent pregnancies. The American journal of cardiology 1997; 80: 1469-1473

28. Sakai K, Imaizumi T, Maeda H et al. Venous distensibility during pregnancy. Comparisons between normal pregnancy and preeclampsia. Hypertension 1994; 24: 461-466

29. Ngan Kee WD. Prevention of maternal hypotension after regional anaesthesia for caesarean section. Current opinion in anaesthesiology 2010; 23: 304-309 DOI: 10.1097/ACO.0b013e328337ffc6

30. Hanss R IC, Missalla H, Steinfath M, Bein B. Continuous noninvasive blood pressure monitoring during spinal anesthesia for cesarean delivery. European Journal of Anaesthesiology 2010; 27: 166-167

31. Chamos C, Vele L, Hamilton M et al. Less invasive methods of advanced hemodynamic monitoring: principles, devices, and their role in the perioperative hemodynamic optimization. Perioperative medicine 2013; 2: 19 DOI: 10.1186/2047-0525-2-19

32. Minville V, Asehnoune K, Salau S et al. The effects of spinal anesthesia on cerebral blood flow in the very elderly. Anesth Analg 2009; 108: 1291-1294 DOI: 10.1213/ane.0b013e31819b073b

33. Obasuyi BI, Fyneface-Ogan S, Mato CN. A comparison of the haemodynamic effects of lateral and sitting positions during induction of spinal anaesthesia for caesarean section. Int J Obstet Anesth 2013; 22: 124-128 DOI: 10.1016/j.ijoa.2012.12.005

34. Klohr S, Roth R, Hofmann T et al. Definitions of hypotension after spinal anaesthesia for caesarean section: literature search and application to parturients. Acta Anaesthesiol Scand 2010; 54: 909-921 DOI: 10.1111/j.1399-6576.2010.02239.x

35. Wakeling HG, McFall MR, Jenkins CS et al. Intraoperative oesophageal Doppler guided fluid management shortens postoperative hospital stay after major bowel surgery. British journal of anaesthesia 2005; 95: 634-642 DOI: 10.1093/bja/aei223

36. Stoneham MD, Eldridge J, Popat M et al. Oxford positioning technique improves haemodynamic stability and predictability of block height of spinal anaesthesia for elective caesarean section. Int J Obstet Anesth 1999; 8: 242-248

37. El-Hakeem EE, Kaki AM, Almazrooa AA et al. Effects of sitting up for five minutes versus immediately lying down after spinal anesthesia for Cesarean delivery on fluid and ephedrine requirement; a randomized trial. Can J Anaesth 2011; 58: 1083-1089 DOI: $10.1007 / \mathrm{s} 12630-011-9593-4$

38. Kim JT, Shim JK, Kim SH et al. Trendelenburg position with hip flexion as a rescue strategy to increase spinal anaesthetic level after spinal block. Br J Anaesth 2007; 98: 396-400 DOI: 10.1093/bja/ael370

39. Racle JP, Benkhadra A, Poy JY et al. Spinal analgesia with hyperbaric bupivacaine: influence of age. Br J Anaesth 1988; 60: 508-514

40. Veering BT, Burm AG, Vletter AA et al. The effect of age on systemic absorption and systemic disposition of bupivacaine after subarachnoid administration. Anesthesiology 1991; 74: 250-257

41. Vercauteren MP, Coppejans HC, Hoffmann VL et al. Small-dose hyperbaric versus plain bupivacaine during spinal anesthesia for cesarean section. Anesth Analg 1998; 86: 989-993

42. Fan SZ, Susetio L, Wang YP et al. Low dose of intrathecal hyperbaric bupivacaine combined with epidural lidocaine for cesarean section--a balance block technique. Anesth Analg 1994; 78: 474-477

43. Van de Velde M, Van Schoubroeck D, Jani J et al. Combined spinal-epidural anesthesia for cesarean delivery: dose-dependent effects of hyperbaric bupivacaine on maternal hemodynamics. Anesth Analg 2006; 103: 187-190, table of contents DOI: $10.1213 / 01$. ane.0000220877.70380.6e

44. Ben-David B, Miller G, Gavriel R et al. Low-dose bupivacaine-fentanyl spinal anesthesia for cesarean delivery. Reg Anesth Pain Med 2000; 25: 235-239

45. Wood RJ, White SM. Anaesthesia for 1131 patients undergoing proximal femoral fracture repair: a retrospective, observational study of effects on blood pressure, fluid administration and perioperative anaemia. Anaesthesia 2011; 66: 1017-1022 DOI: 10.1111/j.13652044.2011.06854.x

46. Cesur M, Alici HA, Erdem AF et al. Spinal anesthesia with sequential administration of plain and hyperbaric bupivacaine provides satisfactory analgesia with hemodynamic stability in cesarean section. Int J Obstet Anesth 2008; 17: 217-222 DOI: 10.1016/j. ijoa.2007.11.007

47. McNaught AF, Stocks GM. Epidural volume extension and low-dose sequential combined spinal-epidural blockade: two ways to reduce spinal dose requirement for caesarean section. Int J Obstet Anesth 2007; 16: 346-353 DOI: 10.1016/j.ijoa.2007.03.013

48. Martyr JW, Stannard KJ, Gillespie G. Spinal-induced hypotension in elderly patients with hip fracture. A comparison of glucose-free bupivacaine with glucose-free bupivacaine and fentanyl. Anaesth Intensive Care 2005; 33: 64-68

49. Veering BT, Ter Riet PM, Burm AG et al. Spinal anaesthesia with $0.5 \%$ hyperbaric bupivacaine in elderly patients: effect of site of injection on spread of analgesia. Br J Anaesth 1996; 77: 343-346

50. Kim JT, Lee JH, Cho CW et al. The influence of spinal flexion in the lateral decubitus position on the unilaterality of spinal anesthesia. Anesth Analg 2013; 117: 1017-1021 DOI: 10.1213/ANE.0b013e3182a1ee53

51. Borodiciene J, Gudaityte J, Macas A. Lithotomy versus jack-knife position on haemodynamic parameters assessed by impedance cardiography during anorectal surgery under low dose spinal anaesthesia: a randomized controlled trial. BMC Anesthesiol 2015; 15: 74 DOI: $10.1186 / \mathrm{s} 12871-015-0055-3$ 
52. Lawicka M, Malek A, Antczak D et al. Non-invasive haemodynamic measurements with Nexfin predict the risk of hypotension following spinal anaesthesia. Anaesthesiol Intensive Ther 2015; 47: 303-308 DOI: 10.5603/AIT.2015.0048

53. Banerjee A, Stocche RM, Angle P et al. Preload or coload for spinal anesthesia for elective Cesarean delivery: a meta-analysis. Can J Anaesth 2010; 57: 24-31 DOI: 10.1007/s12630-009-9206-7

54. Madi-Jebara S, Ghosn A, Sleilaty G et al. Prevention of hypotension after spinal anesthesia for cesarean section: $6 \%$ hydroxyethyl starch 130/0.4 (Voluven) versus lactated Ringer's solution. J Med Liban 2008; 56: 203-207

55. Owczuk R, Wenski W, Polak-Krzeminska A et al. Ondansetron given intravenously attenuates arterial blood pressure drop due to spinal anesthesia: a double-blind, placebo-controlled study. Reg Anesth Pain Med 2008; 33: 332-339 DOI: 10.1016/j.rapm.2008.01.010

56. Liu Y, Pian-Smith MC, Leffert LR et al. Continuous measurement of cardiac output with the electrical velocimetry method in patients under spinal anesthesia for cesarean delivery. J Clin Monit Comput 2015; 29: 627-634 DOI: 10.1007/s10877-014-9645-8

57. Lesser JB, Sanborn KV, Valskys R et al. Severe bradycardia during spinal and epidural anesthesia recorded by an anesthesia information management system. Anesthesiology 2003; 99: 859-866

58. Veeser M, Hofmann T, Roth R et al. Vasopressors for the management of hypotension after spinal anesthesia for elective caesarean section. Systematic review and cumulative meta-analysis. Acta Anaesthesiol Scand 2012; 56: 810-816 DOI: 10.1111/j.13996576.2011.02646.x

59. Doherty A, Ohashi Y, Downey K et al. Phenylephrine infusion versus bolus regimens during cesarean delivery under spinal anesthesia: a double-blind randomized clinical trial to assess hemodynamic changes. Anesth Analg 2012; 115: 1343-1350 DOI: 10.1213/ ANE.0b013e31826ac3db

60. Ngan Kee WD, Lee A, Khaw KS et al. A randomized double-blinded comparison of phenylephrine and ephedrine infusion combinations to maintain blood pressure during spinal anesthesia for cesarean delivery: the effects on fetal acid-base status and hemodynamic control. Anesth Analg 2008; 107: 1295-1302 DOI: 10.1213/ane.0b013e31818065bc

61. Lee A, Ngan Kee WD, Gin T. A quantitative, systematic review of randomized controlled trials of ephedrine versus phenylephrine for the management of hypotension during spinal anesthesia for cesarean delivery. Anesth Analg 2002; 94: 920-926, table of contents

62. Clark DI, Ahmed AB, Baxendale BR et al. Cardiac output during hemiarthroplasty of the hip. A prospective, controlled trial of cemented and uncemented prostheses. J Bone Joint Surg Br 2001; 83: 414-418

63. Ilies C, Kiskalt H, Siedenhans D et al. Detection of hypotension during Caesarean section with continuous non-invasive arterial pressure device or intermittent oscillometric arterial pressure measurement. Br J Anaesth 2012; 109: 413-419 DOI: 10.1093/bja/aes224

64. Jeleazcov C, Krajinovic L, Munster T et al. Precision and accuracy of a new device (CNAPTM) for continuous non-invasive arterial pressure monitoring: assessment during general anaesthesia. Br J Anaesth 2010; 105: 264-272 DOI: 10.1093/bja/aeq143

65. El-Hamamsy I, Durrleman N, Stevens LM et al. Incidence and outcome of radial artery infections following cardiac surgery. Ann Thorac Surg 2003; 76: 801-804

66. Xiao W, Duan QF, Fu WY et al. Goal-directed Fluid Therapy May Improve Hemodynamic Stability of Parturient with Hypertensive Disorders of Pregnancy Under Combined Spinal Epidural Anesthesia for Cesarean Delivery and the Well-being of Newborns. Chin Med J (Engl) 2015; 128: 1922-1931 DOI: 10.4103/0366-6999.160546

67. Bliacheriene F, Carmona MJ, Barretti Cde F et al. Use of a minimally invasive uncalibrated cardiac output monitor in patients undergoing cesarean section under spinal anesthesia: report of four cases. Rev Bras Anestesiol 2011; 61: 610-618, 334-618 DOI: 10.1016/ S0034-7094(11)70072-1

68. McCarthy T, Telec N, Dennis A et al. Ability of non-invasive intermittent blood pressure monitoring and a continuous non-invasive arterial pressure monitor (CNAP) to provide new readings in each 1-min interval during elective caesarean section under spinal anaesthesia. Anaesthesia 2012; 67: 274-279 DOI: 10.1111/j.1365-2044.2011.06996.x 